\title{
Influence of Applied Voltage and Temperature on the Current through the Alumina-filled poly(ethylene-co-butyl acrylate) Nanocomposites Under Constant Stress
}

\author{
N. Jaeverberg, B. Venkatesulu, B.L.G. Jonsson and H. Edin \\ KTH Electrical Engineering \\ Teknikringen 33 \\ SE - 10044 \\ Stockholm, Sweden
}

\begin{abstract}
The current through the poly(ethylene-co-butyl acrylate) with $13 \mathrm{wt} \%$ of butyl acrylate (EBA) filled with $6 \mathrm{wt} \%$ of alumina nanoparticles with an approximate diameter of $45 \mathrm{~nm}$ was studied under constant stress application. The particles were either unmodified or surface-treated with aminopropyltriethoxy silane. The current was measured either until breakdown or for maximum stress duration of 10 minutes. Two voltage levels were chosen: 15 and $22.5 \mathrm{kV}$, which were low enough not to cause a breakdown during the ramping of the voltage at the rate of $490 \mathrm{~V} / \mathrm{s}$ to the constant voltage level at room temperature. Several ramping rates $\{100,260,500$, $1007\} \mathrm{V} / \mathrm{s}$ were also tested for the reference unfilled material to evaluate its influence on the time to breakdown. Several temperature levels were tried: 20 (room temperature), 40 and $50^{\circ} \mathrm{C}$.

As expected, higher applied voltage caused higher current through the materials, while higher temperature caused earlier breakdown as well as higher current. At the lower voltage level most of the materials survived the testing. At the higher voltage level most of the samples of the unfilled reference material failed at the temperatures above room temperature while most of the nanocomposite samples survived the testing even at higher temperatures.
\end{abstract}

\section{Introduction}

The subject of nanocomposite materials was and still is widely discussed in the literature in the recent years [1 5]. A number of material properties such as treeing lifetime and partial discharge resistance were found to be improved by nanostructuration of a material, while some other properties such as breakdown strength as well as dielectric permittivity and loss could either increase, decrease or stay the same as for the reference unfilled material [4]. Understanding the reason behind the latter uncertainties could be advantageous for the ultimate goal with the nanocomposite materials, namely, tailoring of the material properties with nanostructuration of polymeric materials.

In this study the influence of applied voltage and temperature on the current through a nanocomposite based on EBA filled with alumina nanoparticles was evaluated.

\section{Materials}

A detailed description of the materials and sample processing is presented in $[6,7]$. It can be summarized as follows. The unfilled reference material poly(ethylene-co-butyl acrylate) with 13 wt \% of butyl acrylate content, supplied by Borealis (Stenungsund, Sweden). The latter was used either with (REF) or without (REFNAO) the addition of antioxidant Irganox 1010 (a hindered phenolic antioxidant), supplied by Ciba Specialty Chemicals (Germany). The nanocomposite materials were filled with $6 \mathrm{wt} \%$ of the alumina nanoparticles, consisting of $70 \% \delta$-phase and $30 \% \gamma$-phase particles, supplied by Nanophase Inc., USA. These nanoparticles were spherical shape with the average particle diameter of $45 \mathrm{~nm}$ and specific surface area of $36 \mathrm{~m}^{2} / \mathrm{g}$. The nanoparticles could be used either as received (NDU6 materials) or surface-treated with the amino-propyltriethoxy silane (NDA6 materials). The amino-silane was supplied by Sigma-Aldrich. All materials and chemicals were used as received.

All the nanocomposites were manufactured at the Department of Fibre and Polymer Technology, KTH [6].

The samples used in this study had the approximate thickness of $140 \mu \mathrm{m}$ and a diameter of $3 \mathrm{~cm}$. All samples were thoroughly dried in vacuum at $50{ }^{\circ} \mathrm{C}$ for $48 \mathrm{~h}$, followed by $60^{\circ} \mathrm{C}$ for $24 \mathrm{~h}$ prior to testing.

\section{Measurement}

In this study a modified constant stress test [8] was employed. A constant voltage was applied to a sample and the current was measured either until breakdown or for maximum 10 minutes. The duration of the voltage application is chosen such that it exceeds the theoretical dielectric relaxation time constant of the nanocomposite [9]. Two voltage levels were chosen: 15 and $22.5 \mathrm{kV}$, which were low enough not to cause breakdown at room temperature during ramping up the voltage at the rate of $490 \mathrm{~V} / \mathrm{s}$ to the constant voltage level. Several ramping rates $\{100,260,490,1007\} \mathrm{V} / \mathrm{s}$ were also tested to see if it does have an influence on the time to breakdown and charge dynamics in different materials. Several temperature levels were tried: room temperature, 40 and $50{ }^{\circ} \mathrm{C}$. The same measurement cell as in [10] was used and the measurement circuit can be seen in Fig. 1. The 


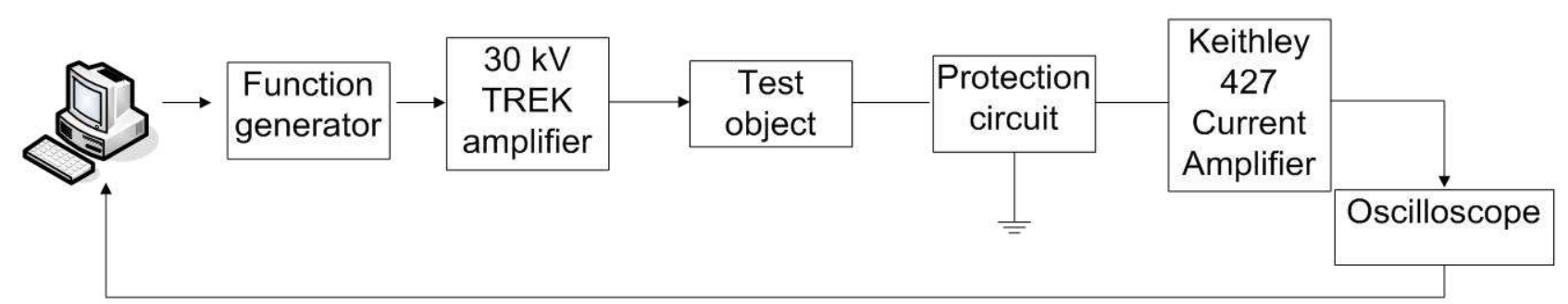

Fig. 1 - Measurement circuit for constant stress testing

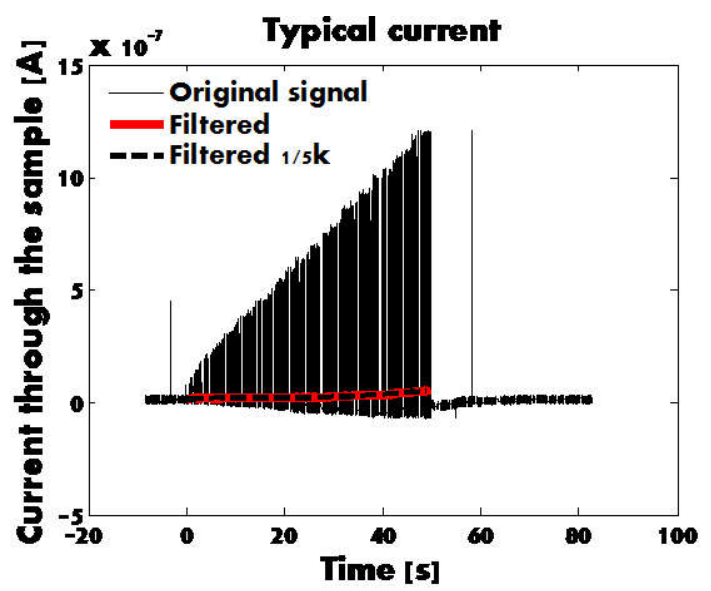

Fig. 2 - An example of a typical original noisy current signal and the filtered curve

electrodes were made of stainless steel with the diameter of $6 \mathrm{~mm}$ and edge radius of $1 \mathrm{~mm}$. All the electrodes were thoroughly cleaned with isopropanol and checked under a microscope for defects prior to testing.

The current through each sample was measured using a Keithley 427 Current Amplifier. Measurements were performed with the lowest filter rise time of the current amplifier of $0.01 \mathrm{~ms}$. This should have allowed registering fast changes in the current but as a consequence the output was very noisy. Filtering was performed in matlab using Savitzky-Golay smoothing filter [11], which is typically used for smoothing out signals with high frequency content. This filter is based on a least squares fit to each window of data by a polynomial of a fixed degree. For this study the window size was chosen to be 3001 points and the polynomial degree was set at 7 . Filtering resulted in a reasonably smooth curve; see Fig. 2 for an example of the original noisy current signal and the filtered curve. Furthermore, it was decided to reduce the number of data points by taking only 1 data point per 5000 existing points for easier plotting, as shown in Fig. 2.

It has to be mentioned here that reassuringly no direct correlation was established between the order in which the samples were tested and the measured current.

\section{Results and discussion}

The speed of ramping up the test voltage at temperatures above room temperature had a strong impact on survival of the reference unfilled samples during the testing. For high ramping speeds the samples failed either during ramp or shortly thereafter, see Fig. 3. There is a clear tendency of increasing current through the samples with increasing temperature for all the tested materials. The same tendency was reported in the literature for LDPE films [12]. Another tendency reported in the literature [13] is the time to breakdown decreasing linearly with increasing temperature under DC applied electric field. In this study it was found that the time to breakdown decreases with increasing temperature, see Table 1 for the data for the unfilled reference material. Statistically speaking the sample size was, however, insufficient in order to confirm linearity of this dependency.

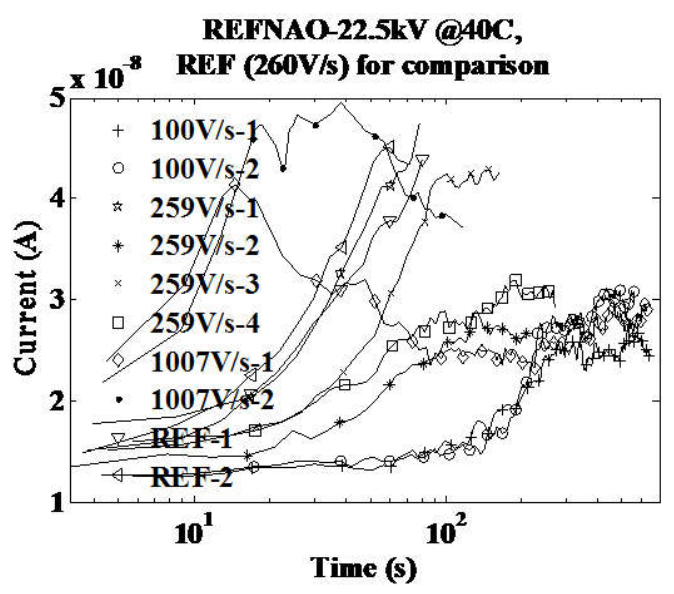

Fig. 3 - Influence of ramping speed on the survival of the reference unfilled material without antioxidant, result for two samples of reference unfilled material with antioxidant at 260 $\mathrm{kV} / \mathrm{s}$ ramping rate is shown for comparison. The legend indicates the ramp in $\mathrm{V} / \mathrm{s}$ - sample number.

Table 1 - Time to breakdown for the reference unfilled material under a DC stress of $22.5 \mathrm{kV}$

\begin{tabular}{|c|c|}
\hline Temperature & Time to breakdown [s] \\
\hline RT & $>600$ \\
\hline $40^{\circ} \mathrm{C}$ & 110 \\
\hline $50^{\circ} \mathrm{C}$ & 45 \\
\hline
\end{tabular}

Considering the stability of the materials at temperatures above the room temperature it can be seen that at the voltage level of $15 \mathrm{kV}$ all materials survive testing, while at voltage level of $22.5 \mathrm{kV}$ it has to be noted that the materials filled with nanoparticles are more reliable than the unfilled materials, see Fig. 4. In fact at $22.5 \mathrm{kV}$ all the samples of the unfilled reference material fail at the temperatures above room temperature, see Fig. 4(b). Unexpectedly, samples of the unfilled reference material without antioxidant fail both 
at room temperature and at $40{ }^{\circ} \mathrm{C}$, while surviving the testing at $50{ }^{\circ} \mathrm{C}$, see Fig. 4(d). On the other hand, only one sample per NDU6 and NDA6 nanocomposites failed testing at $22.5 \mathrm{kV}$ DC voltage level for the temperatures above room temperature (both cases at 50 $\left.{ }^{\circ} \mathrm{C}\right)$, see Fig. 4(f, h). A possible explanation for the failure of the reference unfilled material could be the mechanism involving space charge accumulation [14] which changes the electric field in the material. This explanation is based on existence of zones with different conductivities due to the injected charge travelling through the material and filling up the available traps on its way, which results in a more conducting zone where all the traps are filled. So that the stability of the nanocomposite materials could possibly be explained by higher conductivity in comparison to the unfilled reference material which results in lower space charge accumulation and consequently longer time until breakdown in the same conditions.

Comparing the current through the nanofilled materials it can be said that the current through the materials filled with the untreated nanoparticles was higher than for the materials filled with the nanoparticles surface-treated with the amino-silane, see Fig. 4(e-h). This could indicate that the latter material is more stable than the former, possessing higher breakdown strength as was seen in [10]. Also the behavior of current decay is different between the two nanofilled materials: for the materials filled with the untreated nanoparticles there is an obvious decay in current magnitude with time after reaching a peak, which is somewhat similar to the trend exhibited by the unfilled reference material, see Fig. 4(eh). For the materials filled with the nanoparticles with amino-silane surface treatment the current seems to increase and then stays rather constant, see Fig. 4(g, h). This could be explained by the reactivity of the amino silane surface treatment of the nanoparticles which could capture charges and result lower currents in comparison to other nanocomposites as was shown in [10].

\section{Conclusions}

It was found that higher applied voltage resulted in higher currents through the materials, and higher temperature led to shorter time to breakdown as well as higher current. At the voltage level of $15 \mathrm{kV}$ most of the materials survived the testing. At the voltage level of $22.5 \mathrm{kV}$ most of the samples of the unfilled reference material failed at the temperatures above room temperature while most of the nanocomposite samples survived the testing even at higher temperatures. This could be explained by space charge accumulation which modifies the electric field in the material. The better performance of the nanocomposite materials could be due to higher conductivity in comparison to the reference unfilled material which results in lower space charge.

\section{References}

[1] L. Hui, J.K. Nelson and L.S. Schadler, "Hydrothermal aging of XLPE/silica nanocomposites", Annual Report Conference on Electrical Insulation and Dielectric Phenomena, 2010, pp. $1-4$

[2] J.C. Fothergill, "Ageing, Space Charge and Nanodielectrics: Ten Things We Don't Know About Dielectrics", International Conference on Solid Dielectrics, 2007, pp. 1 - 10

[3] T.J. Lewis "Interfaces are the dominant feature of dielectrics at the nanometric level", IEEE Transactions on Dielectrics and Electrical Insulation, Vol. 11, No. 5, 2004, pp. 739 - 753

[4] T. Tanaka, "Nanodielectrics: how does the presence of interfaces influence behavior?", International Conference on Power and Energy Systems (ICPS), 2011, pp. $1-4$

[5] "Nanodielectrics: a panacea for solving all electrical insulation problems?" International Conference on Solid Dielectrics, 2010

[6] P. Nordell, "Aluminium oxide - poly(ethylene-cobutyl acrylate) nanocomposites: synthesis, structure, transport properties and long-term performance", Lic. Thesis in Polymer Technology, KTH, Sweden, 2011.

[7] N. Jäverberg, H. Edin, P. Nordell, S. Nawaz, B. Azhdar and U. W. Gedde, " Dielectric Properties of Alumina-filled poly(ethylene-co-butyl acrylate) Nanocomposites, part I - dry studies", IEEE Transactions on Dielectrics and Electrical Insulation, Vol. 19, No. 2, 2012, pp. $383-390$

[8] IEC 60243-1, "Electrical Strength of Insulating Materials - Test Methods", International Electrotechnical Commission, 1998

[9] B. Venkatesulu, B.L.G. Jonsson, H. Edin and M. Norgren, "Modeling of insulating nanocompositeselectric and temperature fields", IEEE Transactions on Dielectrics and Electrical Insulation, Vol. 20, No. 1, 2013, pp. 177 - 184

[10] N. Jaeverberg, B. Venkatesulu, and H. Edin, "Dielectric Breakdown Strength of Alumina Filled Poly (ethylene-co-butyl acrylate) Nanocomposites", Conference of Electrical Insulation and Dielectric Phenomena, 2012, pp. 323 - 326

[11] W.H. Press, S.A. Teukolsky, W.T. Vetterling, and B.P. Flannery, Numerical recipes in $C$ : the art of scientific computing, 1993

[12] M. Fukuma, M. Nagao, M. Kosaki, and Y. Kohno, "Measurements of Conduction Current and Electric Field Distribution up to Electrical Breakdown in Two-Layer Polymer Film", Conference of Electrical Insulation and Dielectric Phenomena, 2000, pp. 721-724.

[13] Y. Kishi, H. Miyake, Y. Tanaka, and T. Takada, "Relationship between Breakdown and Space charge Formation in Polyimide Film under DC High Stress", Conference of Electrical Insulation and Dielectric Phenomena, 2009, pp. 146-149. 
[14] K. Matsui, Y. Tanaka, T. Takada, and T. Maeno, "Numerical Analysis of Packet-like Charge Behavior in Low-density Polyethylene under DC
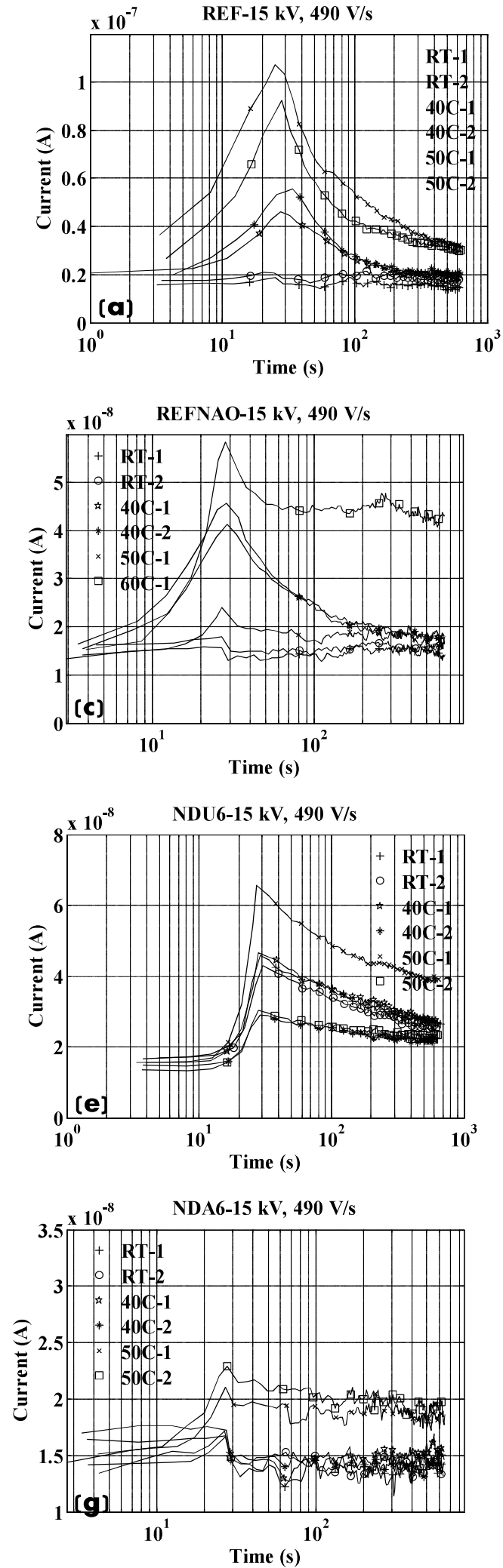

High Electric Field", IEEE Transactions on Dielectrics and Electrical Insulation, Vol. 15, No. 3, 2008, pp. 841-850.
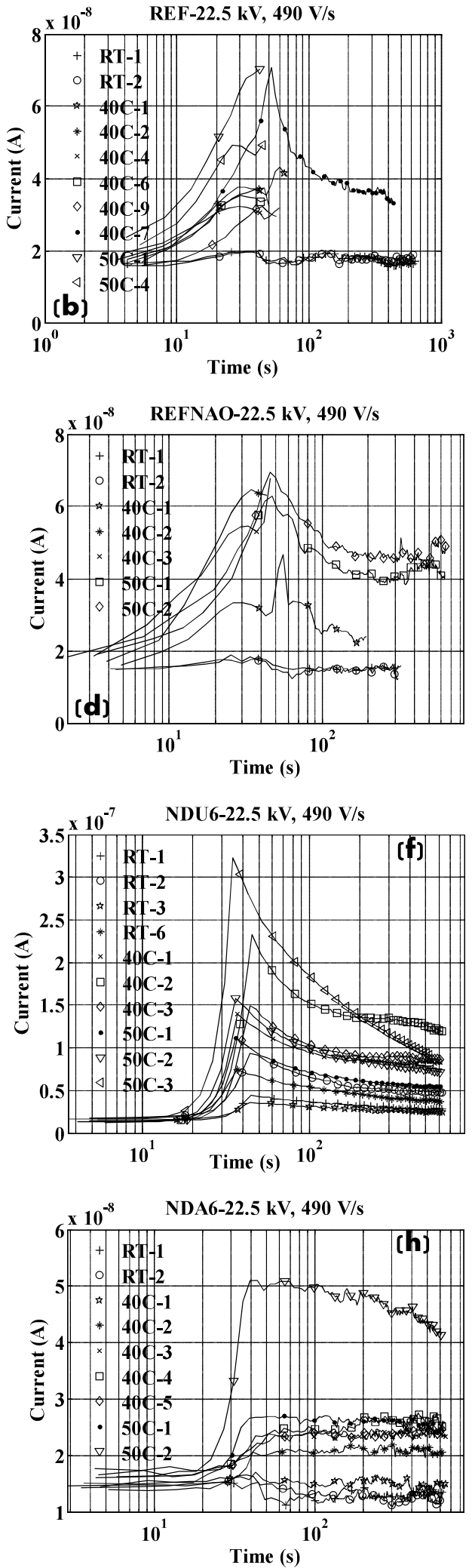

Fig. 4 - Measured current through (a, b) - reference unfilled materials with antioxidants, $(c, d)$ - reference unfilled materials without antioxidants, $(\mathrm{e}, \mathrm{f})$ - materials filled with untreated nanoparticles and $(\mathrm{g}, \mathrm{h})$ materials filled with amino-silane surface-modified nanoparticles. DC voltage stress used was $15 \mathrm{kV}$ for the figures in the left column and $22.5 \mathrm{kV}$ for the results in the right column. The legend indicates the temperature in ${ }^{\circ} \mathrm{C}$ - sample number. 